\section{Minimum performance level definition for bone plate test- ing according to standard: A preliminary study}

\author{
Federico A. Bologna,1,2 Mara Terzini,1,2 \\ Andrea T. Lugas, 1,2 \\ Alberto L. Audenino 1,2 \\ 1Department of Mechanical and \\ Aerospace Engineering, Politecnico di \\ Torino, Turin; ${ }^{2}$ PolitoBIOMed Lab, \\ Politecnico di Torino, Turin, Italy
}

\begin{abstract}
In silico modeling of osteosynthesis medical devices allows the reduction of the time required for experimental tests and the introduction of "simulation-driven design". Using a wise combination of these techniques and analytical calculations, it is possible to relate the experimental results, which are mandatory for regulatory purposes, to the plate physiological application and prevent the occurrence of complications in the early stages after the orthopedic device implantation on humans and animals.
\end{abstract}

\section{Introduction}

Bone plates need to be mechanically tested before commercialization: for this purpose, manufacturers apply test methods published as ISO or ASTM standards. ASTM F384 is the latest and most widely used test standard for metallic angled orthopedic fracture fixation devices. ${ }^{1}$ This specification establishes consistent methods to define the geometric and performance characteristics of bone plates, but it does not require simulating the physiological biomechanics for a specific anatomical region.

Indeed, ASTM standards do not define acceptable levels of performance as, to date, insufficient knowledge is available to predict the consequences of using an osteosynthesis device in individual patients in the first few days after surgery. In this work, analytical and in silico models of physiological conditions are proposed in order to define a minimum performance level to be compared with experimental results obtained with the ASTM F384 setup.

\section{Materials and Methods}

The best and more direct way to establish if the behavior of a plate can guarantee a clinical performance, that can ensure efficacy and safety for the patient, is to compare the estimated fatigue strength, expressed with the maximum bending moment $\left(M_{A S T M}\right)$ that the plate can express while overcoming 1000000 cycles (according to ASTM F384, Figure 1), with the analytically-determined maximum bending moment $\left(M_{\text {Physio }}\right)$ to which the plate is subjected in physiological condition. A "proximal femur plate - femur bone" construct was considered.

First, an analytical model based on the plate critical cross-section has been developed: the plate was positioned on the femur to predict the behavior in correspondence with its critical section when physiological loads were applied. The main reference for the femoral forces was the database 'HIP98'2 from which the reaction on the hip during walking ( $45 \%$ of the step cycle) and muscle forces was extrapolated. Plate performance was evaluated with different cortical bone quality: at 3\%, worst-case selection, and $30 \%$, that represents a more suitable and realistic case scenario.

Quasi-static compression bending tests were then performed to measure the compression bending strength. Set-up configuration was designed to adapt to the specific plate geometry (Figure 1a). Four bone plates were tested to give statistical consistency of results. Exploiting the same set-up, six more plates were tested dynamically to determine the plate fatigue life and the related Maximum Bending Moment. Each test (performed at $5 \mathrm{~Hz}$ ) was ended when either a functional failure was attained, or the test reached 1000000 cycles and repeating the "runout" situation on three different samples.

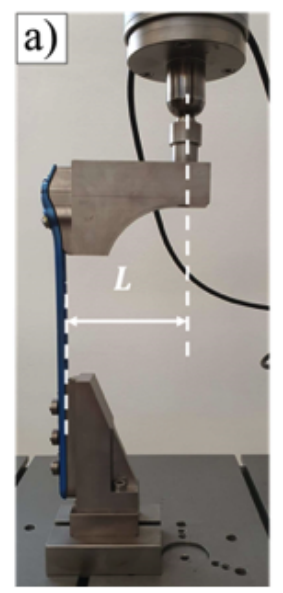

b)

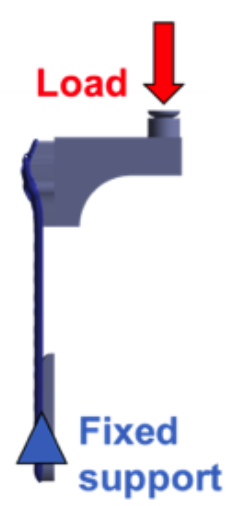

Correspondence: Federico A. Bologna, Department of Mechanical and Aerospace Engineering, Politecnico di Torino, Turin; PolitoBIOMed Lab, Politecnico di Torino, Turin, Italy.

E-mail: federico.bologna@polito.it

Key words: Bone plates; mechanical testing; ASTM F384; finite element analysis.

Disclosures: The authors declare no conflict of interests.

Funding: This research was funded by MIUR, PRIN 2017 BIONIC.

Conference presentation: This paper was presented at the Third Centro 3R Annual Meeting - L'era delle 3R: modelli in silico, in vitro e in vivo per promuovere la ricerca traslazionale 30 September - 1 October 2021, Evento online organizzato dal Politecnico di Torino.

Received for publication: 9 July 2021.

Accepted for publication: 7 September 2021.

This work is licensed under a Creative Commons Attribution NonCommercial 4.0 License (CC BY-NC 4.0).

(C) Copyright: the Author(s), 2021

Licensee PAGEPress, Italy

Biomedical Science and Engineering 2021; 4(s1):154 doi:10.4081/bse.2021.154

Finally, a Finite Element Model (FEM) of the experimental test according to ASTM F384 was implemented (Figure 1b). Plate and set-up materials were considered linear elastic. Tensile properties of plate material (Ti6Al4V) are $E=120 \quad G P a, \sigma_{y}=941.9 \quad M P a$, and $\sigma_{U T S}=991.8 \mathrm{MPa}$, while the anchor blocks were in AISI 630 W1.4542 - Heat Treatment H900. The maximum von Mises stress $\left(\sigma_{\max }\right)$ was positioned on a constant-life fatigue diagram to predict the plate "runout" or rupture.

Figure 1. a) Test bench and b) FEM model, according to ASTM F384. 
cease (blue line delimits the area where no yielding is permitted).

\section{Discussion and Conclusions}

Comparing the most critical bending moment from the analytical model under physiological conditions $\left(M_{\text {Physio }}\right)$ with the maximum bending moment obtained from the experimental tests $\left(M_{A S T M}\right)$ it is possible to demonstrate if the plate is correctly dimensioned for the clinical intended use. In all comparisons between experimental tests and analytical results safety factors $\left(M_{\text {ASTM }} / M_{\text {Physio }}\right)$ were $>3$, thus warranting the safety and effectiveness of the medical device according to its intended purpose. The experimental-virtual comparison for the tested plate confirms the robustness and consistency of both FE model and the Rationale applied.

A further development of the work could be the implementation of an in-silico replica of the femur-plate construct analyzed in the Rationale in order to validate the calculation assumptions.

\section{References}

\section{Results}

The most critical bending moment calculated from the analytical model $\left(M_{\text {Physio }}\right)$ was equal to $18392 \mathrm{Nmm}$ (bone quality $=3 \%$ and body weight=100 kg, Table 1).

The four bone plates tested under quasistatic conditions exhibited plastic deformation, and there were no fractures during the tests. The obtained mean bending strength is equal to $12573.7 \pm 6080.5 \mathrm{Nmm}$. Indeed, from the fatigue tests, three out of six plates exhibited fracture initiating from one of the screw holes, while the others reached the "runout" with an applied bending moment equal to $69228 \mathrm{Nmm}$ (Table 1). Figure 2 shows the von Mises stress state of the plate resultant from the FEM model with a 69228 Nmm bending moment.

Positioning the maximum von Mises stress $(890 \mathrm{MPa})$ on the Haigh diagram for Ti6Al4V 3 it is possible to confirm the obtained results. The maximum stress was in the area under the dotted line: because local yielding is minute, the material may strain-strengthen causing the yielding to
1. ASTM International. ASTM F384-17, Standard Specifications and Test Methods for Metallic Angled Orthopedic Fracture Fixation Devices. ASTM International 2017;1-12.

2. Bergmann G, Graichen F, Rohlmann A. Hip joint loading during walking and running, measured in two patients. J Biomech 1993;26:969-90.

3. Juvinall RC, Marshek KM (eds). Fundamentals of machine component design. 5th Ed., Wiley \& Sons; 2012 